\title{
A novel role for Glucocorticoid-Induced TNF Receptor Ligand (Gitrl) in early embryonic zebrafish development
}

\author{
LYNN D. POULTON ${ }^{1, a, c}$, KATHLEEN F. NOLAN*,1,a, CORINA ANASTASAKI ${ }^{2}$, HERMAN WALDMANN $^{1, b}$ \\ and E. ELIZABETH PATTON $2, b$ \\ ${ }^{1}$ Sir William Dunn School of Pathology, University of Oxford, Oxford, and 2 Institute of Genetics and \\ Molecular Medicine, MRC Human Genetics Unit and The University of Edinburgh, Edinburgh, UK
}

\begin{abstract}
Tumour necrosis factor ligand and receptor superfamily (TNFSF and TNFRSF) members have diverse and well-studied functions in the immune system. Additional, nonimmunological roles, such as in the morphogenesis of bone, tooth, hair and skin have also been described for some members. GITRL and its receptor GITR are well-described as co-regulators of the mammalian immune response. Here, we describe the identification and cloning of their zebrafish homologues and demonstrate a novel role for the ligand, but not the receptor, in early vertebrate development. The assignment of zebrafish Gitrl and Gitr was supported by homology and phylogenetic analysis. The ligand exhibited an oscillating pattern of mRNA expression during the first 36 hours post fertilization, during which time gitr mRNA was not detected, and morpholino oligonucleotide-mediated knock-down of gitrl, but not of gitr, resulted in disruption of early embryogenesis, most clearly revealed during gastrulation, which corresponded to the earliest peak in gitrl mRNA expression (5.25-10 hpf). We found Stat3 signalling to be altered in the gitrl-morphants, suggesting that one possible role for Gitrl during embryogenesis may be modulation of Jak/Stat signalling.
\end{abstract}

KEY WORDS: GITRL, GITR, development, zebrafish, Stat3

\section{Introduction}

The TNF and TNFR superfamilies are receptor-ligand signalling molecules that regulate a variety of pathways, but have primarily been associated with the maintenance and regulation of the immune system. One of these, the glucocorticoid-induced TNF receptor (GITR), in addition to being expressed on activated effector T cells, was also highlighted in a screen for genes expressed by regulatory T cells (Zelenika etal., 2002). Regulatory $T$ cells engage in the suppression of activated effector T cells, including self-reactive $T$ cells, thereby controlling the immune response and helping to prevent autoimmunity. The ligand for GITR, GITRL, is constitutively expressed on antigen presenting cells. Its expression is transiently increased in response to inflammatory stimuli, and engagement of GITR by GITRL leads to both dampening of the suppressive effects of regulatory $T$ cells, and to co-activation of effector $T$ cells (Tone et al., 2003). The net effective outcome is strong activation of the immune response, and indeed, antibody-mediated stimulation of GITR can induce autoimmunity (Shimizu et al., 2002). The potential to modulate immune regulation through GITRL-GITR signalling has made this pathway an interesting candidate for study in cancer immunotherapy and in models of persistent infection.

In mice, targeted disruption of the GITR gene results in T cell hypersensitivity to CD3 activation, leading to hyperproliferation and heightened sensitivity to activation-induced cell death (Ronchetti et al., 2002), as well as altered responses to Candida

\footnotetext{
Abbreviations used in this paper: dpf, days post-fertilization; EDA, ectodysplasin; hpf, hours post fertilization; GITR, glucocorticoid-induced TNF receptor; GITRL, glucocorticoid-induced TNF receptor ligand; MO, morpholino oligonucleotide; TNFSF, tumour necrosis factor superfamily; TNFRSF tumour necrosis factor receptor superfamily.
}

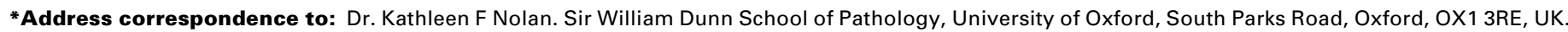
Fax: +44 (0)1865-275501. e-mail: kathleen.nolan@path.ox.ac.uk
}

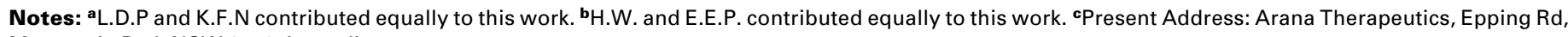
Macquarie Park NSW 2113 Australia 
albicans (Agostini et al., 2005) and in experimentally induced acute lung inflammation (Cuzzocrea et al., 2006). With a specific interest in the role of GITRL in these phenomena, and anticipating an immune phenotype similar to that seen in the Gitrknockout, we generated a mouse knockout of the GITRL gene and were surprised by a strain-dependent embryonic lethal phenotype that suggested an essential role for GITRL in development (manuscript in preparation). To gain specific insight into the developmental requirements for GITRL we turned to the zebrafish system, now widely used as a complementary vertebrate model for studies in both immunology and development.

A role for the TNF/TNFR families in the development of nonlymphoid tissues has precedents. Although initially highlighted for its role in the immune system, RANKL has been shown to be essential for osteoclast differentiation and bone remodelling in mice, as well as lymph-node organogenesis and the early differ-

A

\section{GITRL (TNFSF18)}

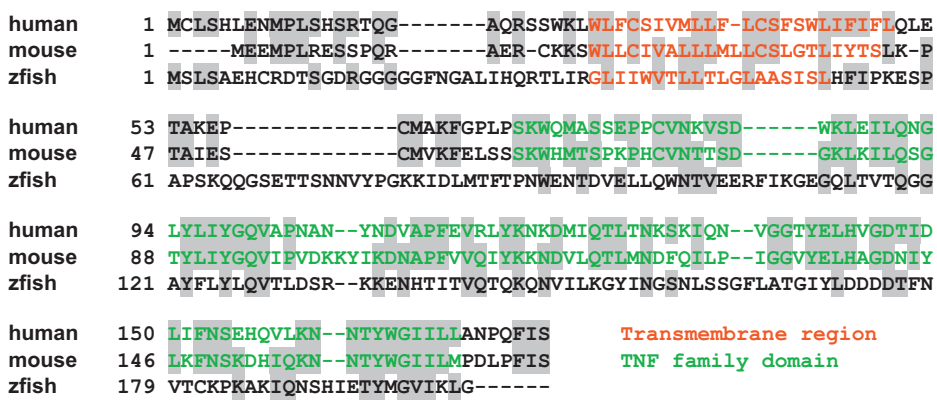

\section{B GITR (TNFRSF18)}

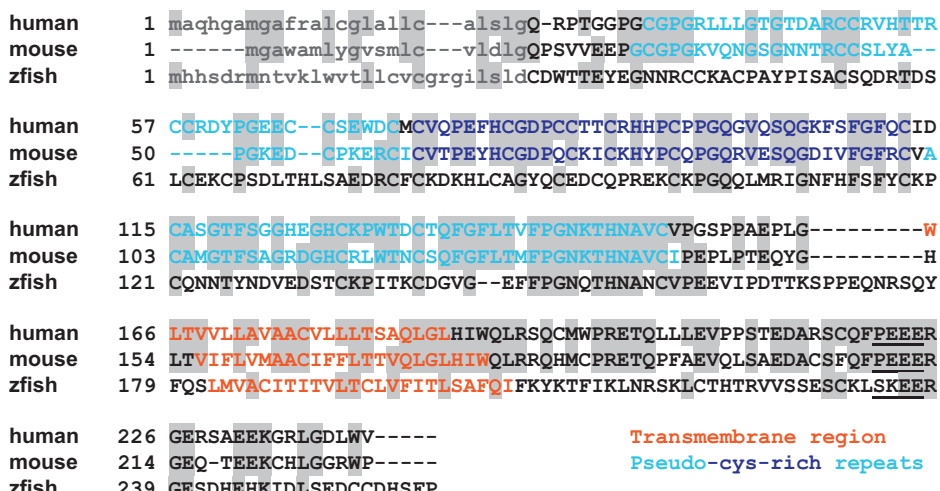

entiation of thymocytes and B cell precursors (Kong et al., 1999), while in humans mutations in RANKL underlie autosomal recessive osteoclast-poor osteopetrosis (Frattini et al., 2007). Ectodysplasin (EDA) is the only TNFSF member to date whose function remains exclusively non-immune. Mutations in EDA in mice (Tabby) affect development of the skin, hair and teeth (Ferguson et al., 1997), and similarly in humans are causative of X-linked anhidrotic ectodermal dysplasia, a disorder characterised by sparse hair, absence of sweat glands and abnormal or missing teeth (Kere et al., 1996).

Here we describe the identification of genes encoding homologues of mammalian GITRL and GITR in zebrafish. We have cloned the corresponding ligand and receptor transcripts and demonstrate, by differential gene expression and morpholino oligonucleotide (MO)-mediated gene knockdown, a critical role for the ligand in early embryonic development. This role is

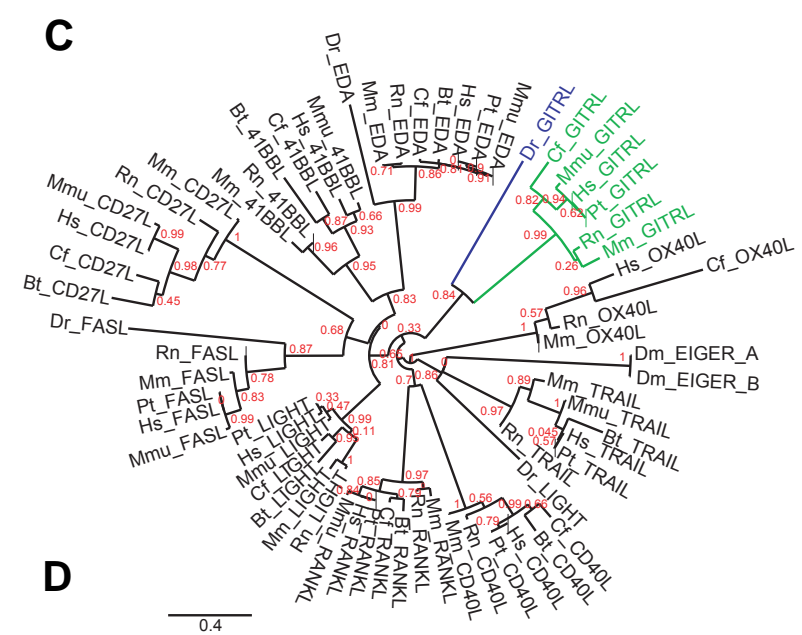

Fig. 1. Alignment and phylogenetic analysis of candidate zebrafish Gitrl and Gitr with species homologues and other TNF/TNFR superfamily members. Alignment of human, mouse and zebrafish (zfish) (A) GITRL and (B) GITR protein sequences are shown with identical residues highlighted by shading. Protein accession numbers are detailed in Tables 1 and 2. Transmembrane regions and the GITR signal sequence (lower case) are annotated as assigned in the Ensemble database entry for mouse and human, and as predicted using TMpred for the zebrafish sequences. The extent of the TNF family domain of GITRL and the three pseudo-cysteine rich domains of GITR are marked as their respective Ensemble and

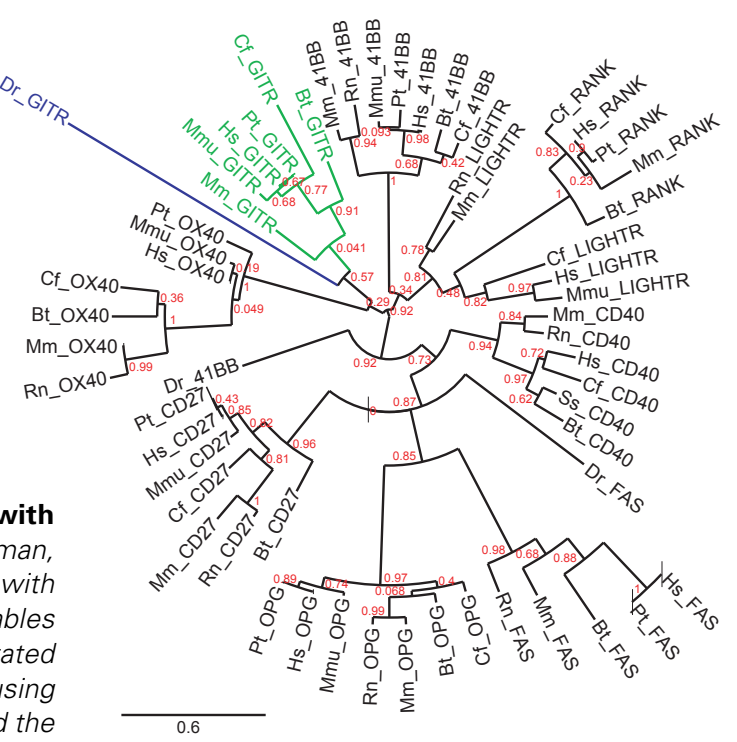
Uniprot database entries. A TRAF-2 binding consensus (P/S/A/T)x(Q/E)E conserved in the cytoplasmic tail of GITR is underlined (Ye et al., 1999). Phylograms generated using selected (C) TNF and (D) TNFR superfamily members confirmed that the cloned zebrafish sequences cluster with mammalian homologues of GITRL and GITR respectively. The analyses were performed using nucleotide coding sequences extracted from database entries detailed in Tables 1 and 2. Branch support based on an approximation of the standard likelihood ratio test is indicated (Dereeper et al., 2008). Synteny information to support the assignment of species homologues was established, except for Dr_GITR, Dr_GITRL, Dr_EDA, Dr_LIGHT and Dr_FasL. 
independent of the cloned Gitr sequence, but is associated with changes in Stat3 phosphorylation.

\section{Results}

\section{Identification and cloning of homologues of GITRL and GITR in zebrafish}

Exons of zebrafish gitrland gitrwere provisionally identified by (Zebrafish Gitrland Gitr Genbank accession numbers, EU099310 and EU099311) amplified and cloned from adult zebrafish kidney. The translated sequences were found to share $17-22 \%$ amino $A, B)$, a level consistent with those previously reported for a number of other fish TNFSF members (Glenney and Wiens, 2007). The human and mouse GITRL and GITR proteins share quently cloned from whole zebrafish embryos to confirm that the embryonic sequence was identical to the adult.

To further establish the assignment of the newly cloned zebrafish TNF and TNF receptor superfamily members, phylogenetic analyses were performed using well-defined species homologues of a selection of ligand and receptor superfamily members, mainly selected on the basis of close homology or by virtue of their

\section{A}

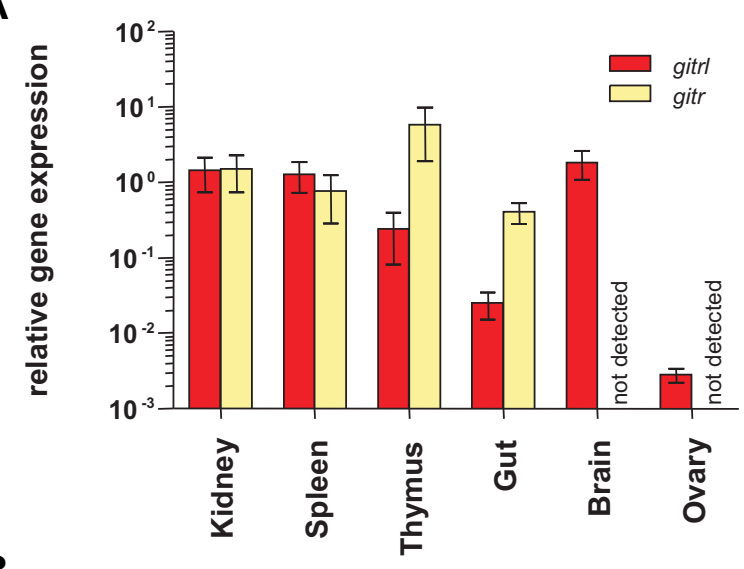

B

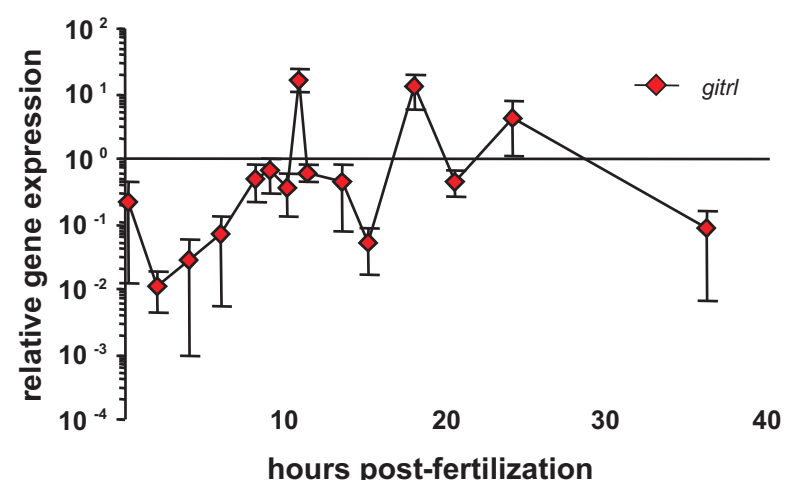
homology using BLAST, and their corresponding transcripts acid identity with their mouse and human counterparts (Fig. 1 only $51-58 \%$ identity. The candidate gitr/cDNA was also subse-

genetic locus. TNF superfamily members with well-described roles in development, RANKL and EDA, and the earliest known TNF family member, EIGER (Moreno et al., 2002), were also included. Both the zebrafish ligand and receptor nucleotide coding sequences grouped appropriately with mammalian species homologues of GITRL and GITR respectively (Fig. 1 C,D)

\section{Gitrl, but not gitr, is expressed during the first two days of embryogenesis}

Using real-time PCR analysis, gitr/and gitrwere both found to be transcribed in the lymphoid tissues of adult zebrafish including the kidney marrow (Fig. 2A). The ligand was additionally detected in non-lymphoid tissues of the ovary and brain (Fig. 2A). During the first 36 hours of embryonic development, the ligand exhibited a multiphasic expression pattern (Fig. 2B). An early peak (0-2 hpf), presumably representing maternal mRNA prior to the start of zygotic transcription, dropped away rapidly. Expression reappeared during gastrulation (5.25-10 hpf), rising to a peak corresponding to the start of segmentation (10.5 hpf). From here, expression dipped as segmentation proceeded, rising again to peak during late segmentation (approx. $18 \mathrm{hpf}$ ) with another, lower, peak at $24 \mathrm{hpf}$ (early pharyngula). This oscillating pattern of expression suggests that Gitrl may act at multiple time points during the first 36 hours of development. In contrast, gitrexpres-

C
$10 \mathrm{hpf}$
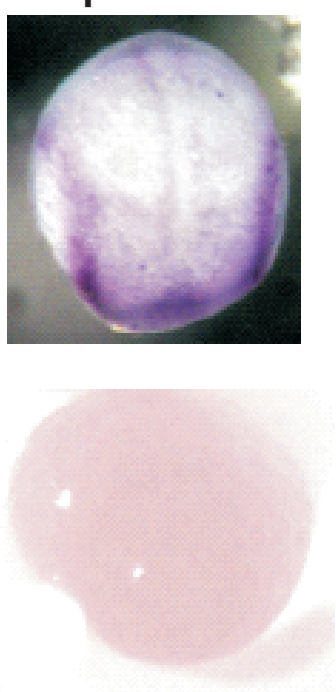

control
$26 \mathrm{hpf}$

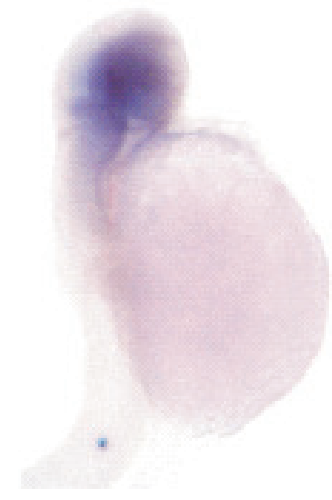

Fig. 2. Expression of zebrafish gitrl and gitr genes in adult tissues and during development. Real-time PCR was used to assess the expression of gitrl and gitr in (A) adult zebrafish tissues and (B) at different time points during embryonic development. Gene expression was quantified using a standard curve method and is shown normalised to ef $1 \alpha$ transcript levels and expressed relative to levels in adult kidney.

Values are shown as mean $\pm S D$ of technical triplicates for an individual experiment representative of three biological replicates. In adult tissues gitr mRNA was not detected in brain or ovary. In embryos, only gitrl is shown as no gitr mRNA was detected during this period. (C) Localisation of gitrl transcripts at different time-points within developing embryos was investigated by in situ hybridization using a DIG-labelled antisense RNA probe. Distinct bands along the paraxial mesoderm can be seen at $10 \mathrm{hpf}$, with localization of expression to the head region by 26 hpf. A control embryo processed at $16 \mathrm{hpf}$ in the absence of probe is shown to indicate the level of background staining and any yolk trapping. 
sion was undetectable during this period.

To assess localization patterns corresponding to the temporal variations described, wild-type embryos were probed for the presence of gitr/ mRNA at various early developmental timepoints using whole-mount in situhybridization. Transcription was ubiquitous during the first $8 \mathrm{hpf}$, with some concentration of expression to the germ ring during early gastrulation (data not shown). In late gastrulation and early segmentation, distinct bands of patterning were evident along the paraxial mesoderm (Fig. 2C, $10 \mathrm{hpf}$ ). By late segmentation, low expression of gitr/was detectable in the anterior of the embryo, which by the midpharyngula period had localised to the head region (Fig. 2C, 26 hpf).

\section{Expression of gitrl, but not gitr, is required during early development}

The patterning of gitrlexpression (Fig. 2B, C) suggested to us that it might have specific functions during embryogenesis. To explore this possibility, we used MOs to knock down gitr/expression and embryos were monitored for the emergence of morphological abnormalities. Although the presence of gitrmRNA had not been detected in the first two days post-fertilization, the possibility of very low, but functionally relevant levels of expression prompted us to test gitr MOs as well. One translation-block (zGL1, zGR1) and one splice-block (zGL2, zGR2) MO were tested for each gene. Both ligand MOs gave a similar dose-dependent mutant embryonic phenotype (Fig. 3A), which was not observed with gitr

TABLE 1

TNFSF MEMBERS USED FOR PHYLOGENETIC ANALYSIS

\begin{tabular}{|c|c|c|c|c|}
\hline Label in tree (Fig.1) & Protein & Species & Transcript accession \# & Protein accession \# \\
\hline Dm_EIGER_A & EIGER, isoform $A$ & Drosophila melanogaster & NM_165735 & NP_724878 \\
\hline Dm_EIGER_B & EIGER, isoform B & Drosophila melanogaster & NM_206069 & NP_995791 \\
\hline Bt_EDA & Ectodysplasin-A & Bos taurus & NM 001081743 & NP_001075212 \\
\hline Cf_EDA & Ectodysplasin-A & Canis lupus familiaris & NM_001014770 & NP_001014770 \\
\hline Hs EDA & Ectodysplasin-A & Homo sapiens & NM_001399 & NP_001390 \\
\hline Mm_EDA & Ectodysplasin-A & Mus musculus & NM_010099 & NP_034229 \\
\hline Mmu_EDA & Ectodysplasin-A & Macaca mulatta & XM_001082424 & XP_001082424 \\
\hline Pt EDA & Ectodysplasin-A & Pan troglodytes & XM 529025 & XP 529025 \\
\hline Rn_EDA & Ectodysplasin-A & Rattus norvegicus & XM_228582 & XP_228582 \\
\hline Cf_OX40L & TNFSF4 & Canis lupus familiaris & XM 547459 & XP_547459 \\
\hline $\mathrm{Hs} \_\mathrm{O} X 40 \mathrm{~L}$ & TNFSF4 & Homo sapiens & NM_003326 & NP_003317 \\
\hline Mm_OX40L & TNFSF4 & Mus musculus & NM_009452 & NP_033478 \\
\hline $\mathrm{Rn} \_\mathrm{OX} 40 \mathrm{~L}$ & TNFSF4 & Rattus norvegicus & NM_053552 & NP_446004 \\
\hline Bt_CD40L & TNFSF5 & Bos taurus & NM_174624 & P51749 \\
\hline $\mathrm{Hs}$ CD40L & TNFSF5 & Homo sapiens & NM 000074 & NP 000065 \\
\hline Mm_CD40L & TNFSF5 & Mus musculus & NM_011616 & NP_035746 \\
\hline $\mathrm{Rn} \_\mathrm{CD} 40 \mathrm{~L}$ & TNFSF5 & Rattus norvegicus & NM_053353 & NP_445805 \\
\hline Dr_FASL & TNFSF6 & Danio rerio & NM_001042701 & NP_001036166 \\
\hline Hs_FASL & TNFSF6 & Homo sapiens & NM_000639 & NP_000630 \\
\hline Mm_FASL & TNFSF6 & Mus musculus & NM_010177 & NP_034307 \\
\hline Mmu_FASL & TNFSF6 & Macaca mulatta & NM_001032838 & NP_001028010 \\
\hline Pt_FASL & TNFSF6 & Pan troglodytes & XM_524967 & XP_524967 \\
\hline Rn_FASL & TNFSF6 & Rattus norvegicus & NM_012908 & NP_037040 \\
\hline Bt_CD27L & TNFSF7 & Bos taurus & XM_600347 & XP_600347 \\
\hline Cf CD27L & TNFSF7 & Canis lupus familiaris & XM 542136 & XP 542136 \\
\hline $\mathrm{Hs}$ _CD27L & TNFSF7 & Homo sapiens & NM_001252 & NP_001243 \\
\hline $\mathrm{Mm}$ CD27L & TNFSF7 & Mus musculus & NM_011617 & NP 035747 \\
\hline Mmū_CD27L & TNFSF7 & Macaca mulatta & XM_001088935 & XP_001088935 \\
\hline $\mathrm{Rn}$ CD27L & TNFSF7 & Rattus norvegicus & NM 001106878 & XP_001059337 \\
\hline Bt_41BBL & TNFSF9 & Bos taurus & XM_600015 & XP_600015 \\
\hline Cf_41BBL & TNFSF9 & Canis lupus familiaris & XM_533933 & XP_533933 \\
\hline $\mathrm{Hs}$ 41BBL & TNFSF9 & Homo sapiens & NM 003811 & NP 003802 \\
\hline Mm_41BBL & TNFSF9 & Mus musculus & NM_009404 & NP_033430 \\
\hline Mmu_41BBL & TNFSF9 & Macaca mulatta & XM_001088828 & XP_001088828 \\
\hline Rn_41BBL & TNFSF9 & Rattus norvegicus & NM_181384 & NP_852049 \\
\hline Bt_TRAIL & TNFSF10 & Bos taurus & XM_583785 & XP_583785 \\
\hline Hs_TRAIL & TNFSF10 & Homo sapiens & NM_003810 & NP_003801 \\
\hline Mm_TRAIL & TNFSF10 & Mus musculus & NM_009425 & NP_033451 \\
\hline Mmu_TRAIL & TNFSF10 & Macaca mulatta & XM_001084768 & XP_001084768 \\
\hline Pt_TRAIL & TNFSF10 & Pan troglodytes & XM_001165714 & XP_001165714 \\
\hline Rn_TRAIL & TNFSF10 & Rattus norvegicus & NM_145681 & NP_663714 \\
\hline Bt_RANKL & TNFSF11 & Bos taurus & XM 591585 & XP_591585 \\
\hline Cf_RANKL & TNFSF11 & Canis lupus familiaris & XM_846672 & XP_851765 \\
\hline Hs_RANKL & TNFSF11 & Homo sapiens & NM_003701 & NP_003692 \\
\hline Mm_RANKL & TNFSF11 & Mus musculus & NM_011613 & NP_035743 \\
\hline Mmu_RANKL & TNFSF11 & Macaca mulatta & XM_001092211 & XP_001092211 \\
\hline Pt_RĀNKL & TNFSF11 & Pan troglodytes & XM_522750 & XP_522750 \\
\hline Rn_RANKL & TNFSF11 & Rattus norvegicus & NM_057149 & NP_476490 \\
\hline Bt LIGHT & TNFSF14 & Bos taurus & NM 001101855 & NP 001095325 \\
\hline Cf_LIGHT & TNFSF14 & Canis lupus familiaris & XM_849235 & XP_854328 \\
\hline Dr_LIGHT & TNFSF14 & Danio rerio & XM_001338537 & XP_001338573 \\
\hline Hs_LIGHT & TNFSF14 & Homo sapiens & NM_003807 & NP_003798 \\
\hline Mm_LIGHT & TNFSF14 & Mus musculus & NM_019418 & NP_062291 \\
\hline Mmu_LIGHT & TNFSF14 & Macaca mulatta & XM_001089169 & XP_001089169 \\
\hline Pt_LIGHT & TNFSF14 & Pan troglodytes & Xm_001149848 & XP_001149848 \\
\hline Rn LIGHT & TNFSF14 & Rattus norvegicus & XM 001059278 & XP 001059278 \\
\hline Cf_GITRL & TNFSF18 & Canis lupus familiaris & XM_848899 & XP_853992 \\
\hline Dr_GITRL & TNFSF18 & Danio rerio & EU099310 & ABV22578 \\
\hline Hs GITRL & TNFSF18 & Homo sapiens & NM 005092 & NP 005083 \\
\hline Mm_GITRL & TNFSF18 & Mus musculus & NM_183391 & NP_899247 \\
\hline Mmu GITRL & TNFSF18 & Macaca mulatta & NM 001040191 & NP 001035281 \\
\hline Pt_GITRL & TNFSF18 & Pan troglodytes & XM_001147996 & XP_001147996 \\
\hline Rn_GITRL & TNFSF18 & Rattus norvegicus & XM_344166 & XP 344167 \\
\hline
\end{tabular}


or control MOs, all of which resulted in apparently normal embryonic development (Fig. 3). This is consistent with our inability to detect gitr message during this period of development. We independently demonstrated that the efficacy of the splice-block gitr/MO persisted for the length of the experimental observation (Fig. 3B), and established that it induced mis-splicing of exon 1 to exon 3 resulting in a frame-shift and the introduction of a premature stop codon, such that any resulting protein would completely lack the TNF region required for multimerisation and receptor binding (Fig. 3C).

The majority of gitr/morphants showed delayed and abnormal gastrulation, abnormal somitogenesis, abnormal neurogenesis, decreased growth and high mortality. From 70-100\% epiboly, morphants had a noticeable developmental lag compared to controls and showed mild perturbations of shape (Fig. $3 \mathrm{E}, \mathrm{J}$ ). It was also noticeable that the blastoderm margin in gitr/morphants did not reach its normal full extent by the end of epiboly (Fig. 3J), and abnormalities were consistently observed by the beginning of segmentation (Fig. 3K). Anterior somites formed, but lacked clear boundaries (Fig. $3 \mathrm{~K}, \mathrm{~L}$ ), and although posterior somites had clearer boundaries, they failed to form the characteristic chevron shape observed in control embryos (Figs. $3 \mathrm{H}, \mathrm{M}$, magnified in Fig. $3 \mathrm{~S}, \mathrm{~T})$. The optic primordium appeared late in morphants and remained small and misshapen (Fig. 3 L,M). Morphants remained consistently smaller and had abnormal yolk extensions (Fig. 3M).

We noticed that the gitr/ morphant embryos had a reduced notochord anlage (data not shown) suggesting possible reduction of cell movements (Kane et al., 1996, Krens et al., 2008). To examine this phenotype more closely, we used the molecular markers $d / x 3$ (the edge neural plate marker) and hggl (the presumptive hatching gland marker) to compare the gitr/morphant phenotypes to control embryos (Krens et al., 2008). The neural plate domain was wider in the gitr/morphants, as indicated by the broadened $d / x 3$ expression domain (Fig. $3 \mathrm{U}, \mathrm{V}$ ), confirming that

TABLE 2

TNFRSF MEMBERS USED FOR PHYLOGENETIC ANALYSIS

\begin{tabular}{|c|c|c|c|c|}
\hline Label in tree (Fig. 1) & Protein & Species & Transcript accession \# & Protein accession \# \\
\hline Bt OX40 & TNFRSF4 & Bos Taurus & NM 001099043 & NP 001092513 \\
\hline Cf OX40 & TNFRSF4 & Canis lupus familiaris & XM_546720 & XP_546720 \\
\hline $\mathrm{Hs} \mathrm{OX} 40$ & TNFRSF4 & Homo sapiens & NM_003327 & NP_003318 \\
\hline $\mathrm{Mm}$ OX40 & TNFRSF4 & Mus musculus & NM 011659 & NP 035789 \\
\hline Mmu_OX40 & TNFRSF4 & Macaca mulatta & XM_001090870 & XP_001090870 \\
\hline Pt OX40 & TNFRSF4 & Pan troglodytes & XM 513705 & XP 513705 \\
\hline Rn_OX40 & TNFRSF4 & Rattus norvegicus & NM_013049 & NP_037181 \\
\hline $\mathrm{Bt}$ CD40 & TNFRSF5 & Bos Taurus & NM 001105611 & NP 001099081 \\
\hline Cf_CD40 & TNFRSF5 & Canis lupus familiaris & NM_001002982 & NP_001002982 \\
\hline $\mathrm{Hs}$-CD40 & TNFRSF5 & Homo sapiens & NM_001250 & NP_001241 \\
\hline $\mathrm{Mm}$ CD40 & TNFRSF5 & Mus musculus & NM 011611 & NP 035741 \\
\hline Rn_CD40 & TNFRSF5 & Rattus norvegicus & NM_134360 & NP_599187 \\
\hline Ss CD40 & TNFRSF5 & Sus scrofa & NM_214194 & NP 999359 \\
\hline Bt_FAS & TNFRSF6 & Bos Taurus & NM_174662 & NP_777087 \\
\hline Dr FAS & TNFRSF6 & Danio rerio & XM 685355 & $X P-690447$ \\
\hline Hs_FAS & TNFRSF6 & Homo sapiens & NM_000043 & NP_000034 \\
\hline Mm_FAS & TNFRSF6 & Mus musculus & NM_007987 & NP_032013 \\
\hline Pt FAS & TNFRSF6 & Pan troglodytes & XM 001139138 & XP 001139138 \\
\hline Rn_FAS & TNFRSF6 & Rattus norvegicus & NM_139194 & NP_631933 \\
\hline $\mathrm{Bt} \mathrm{CD} 27$ & TNFRSF7 & Bos Taurus & NM 001082434 & NP 001075903 \\
\hline Cf_CD27 & TNFRSF7 & Canis lupus familiaris & XM_849371 & XP_854464 \\
\hline $\mathrm{Hs}$ CD27 & TNFRSF7 & Homo sapiens & NM_001242 & NP_001233 \\
\hline $\mathrm{Mm} \_\mathrm{CD} 27$ & TNFRSF7 & Mus musculus & NM_001033126 & NP_001028298 \\
\hline Mmu_CD27 & TNFRSF7 & Macaca mulatta & XM_001104258 & XP_001104258 \\
\hline Pt CD27 & TNFRSF7 & Pan troglodytes & XM 508952 & XP 508952 \\
\hline Rn_CD27 & TNFRSF7 & Rattus norvegicus & NM_001024335 & NP_001019506 \\
\hline Bt_41BB & TNFRSF9 & Bos Taurus & NM_001035336 & NP_001030413 \\
\hline Cf_41BB & TNFRSF9 & Canis lupus familiaris & XM_845243 & XP_850336 \\
\hline $\mathrm{Dr}_{-} 41 \mathrm{BB}$ & TNFRSF9 & Danio rerio & XM_001923079 & XP_001923114 \\
\hline $\mathrm{Hs}$-41BB & TNFRSF9 & Homo sapiens & NM_001561 & NP_001552 \\
\hline $\mathrm{Mm} \_41 \mathrm{BB}$ & TNFRSF9 & Mus musculus & NM_001077509 & NP_001070977 \\
\hline Mmu $41 \mathrm{BB}$ & TNFRSF9 & Macaca mulatta & XM_001096166 & XP 001096166 \\
\hline Pt_41BB & TNFRSF9 & Pan troglodytes & XM_001157732 & XP_001157732 \\
\hline $\mathrm{Rn}$ 41BB & TNFRSF9 & Rattus norvegicus & NM_001025773 & NP_001020944 \\
\hline Bt_RANK & TNFRSF11A & Bos Taurus & XM_609364 & XP_609364 \\
\hline Cf_RANK & TNFRSF11A & Canis lupus familiaris & XM_541077 & XP 541077 \\
\hline $\mathrm{Hs}$ RANK & TNFRSF11A & Homo sapiens & NM 003839 & NP 003830 \\
\hline Mm_RANK & TNFRSF11A & Mus musculus & NM_009399 & NP_033425 \\
\hline Pt RANK & TNFRSF11A & Pan troglodytes & XM 523949 & XP 523949 \\
\hline Bt_OPG & TNFRSF11B & Bos Taurus & NM_001098056 & NP_001091525 \\
\hline Cf_OPG & TNFRSF11B & Canis lupus familiaris & XM_539146 & XP 539146 \\
\hline $\mathrm{Hs}$ OPG & TNFRSF11B & Homo sapiens & NM 002546 & NP 002537 \\
\hline Mm_OPG & TNFRSF11B & Mus musculus & NM_008764 & NP 032790 \\
\hline Mmu OPG & TNFRSF11B & Macaca mulatta & XM 001096915 & XP 001096915 \\
\hline Pt_OPG & TNFRSF11B & Pan troglodytes & XM_519921 & XP_519921 \\
\hline Rn OPG & TNFRSF11B & Rattus norvegicus & NM 012870 & NP 037002 \\
\hline Hs_LIGHTR & TNFRSF14 & Homo sapiens & NM_003820 & NP_003811 \\
\hline Rn_LIGHTR & TNFRSF14 & Rattus norvegicus & NM_001015034 & NP_001015034 \\
\hline $\mathrm{Mm}$ LIGHTR & TNFRSF14 & Mus musculus & NM 178931 & NP 849262 \\
\hline Cf_LIGHTR & TNFRSF14 & Canis lupus familiaris & XM_549666 & XP_549666 \\
\hline Mmu LIGHTR & TNFRSF14 & Macaca mulatta & NM 001043357 & NP 001036822 \\
\hline Bt_GITR & TNFRSF18 & Bos Taurus & XM_594408 & XP_594408 \\
\hline Cf GITR & TNFRSF18 & Canis lupus familiaris & XM 843467 & $X P-848560$ \\
\hline Dr_GITR & TNFRSF18 & Danio rerio & EU099311 & NP_001106996 \\
\hline Hs_GITR & TNFRSF18 & Homo sapiens & NM_004195 & NP_004186 \\
\hline $\mathrm{Mm}$ GITR & TNFRSF18 & Mus musculus & NM 009400 & NP 033426 \\
\hline Mmu_GITR & TNFRSF18 & Macaca mulatta & XM_001090511 & XP_001090511 \\
\hline Pt GITR & TNFRSF18 & Pan troglodytes & XM 001144377 & XP 001144377 \\
\hline
\end{tabular}


A

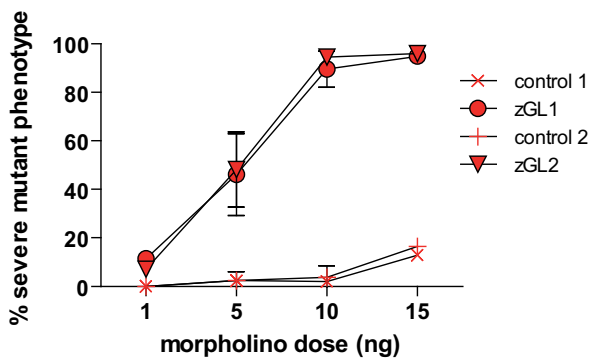

B

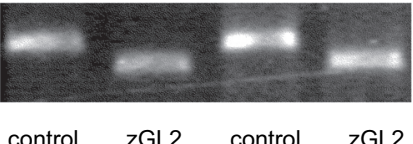

$10 \mathrm{hpf}$
$18 \mathrm{hpf}$
C

control MSLSAEHCRDTSGDRGGGGGFNGALIHORTLIRGLI IWVTLITLGLAAS ISLHFIPKESPAPSKOOG zGL2 MSLSAEHCRDTSGDRGGGGGFNGALIHQRTLIRGLIIWVTLLTLGLAAS ISLHFIPKESPAPSKQQG

control SETTSNNVYPGKKIDLMTFTPNWENTDVELLQWNTVEERFIKGEGQLTVTQGGAYFLYLQVTLDSRK

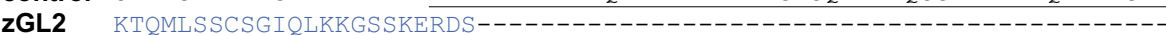

control KENHTITVQTQKQNVILKGYINGSNLSSGFLATGIYLDDDDTFNVTCKPKAKIQNSHIETYMGVIKLG zGL2
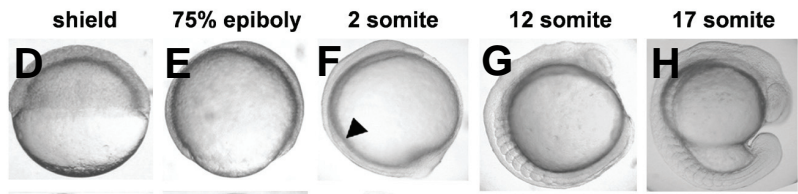

control
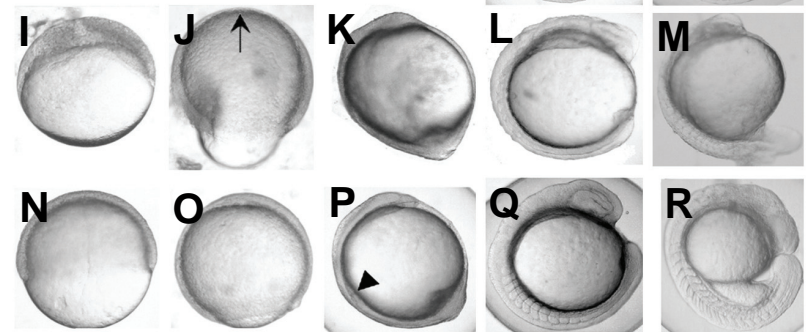

zGR

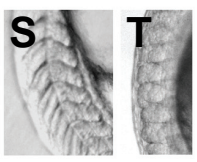

control zGL2

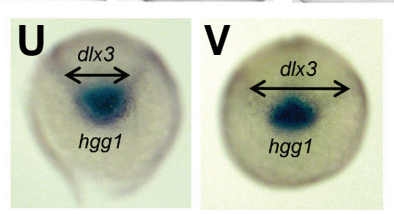

Fig. 3. Morpholino oligonucleotide knock-down of gitrl during early development. (A) Embryos injected with increasing amounts of zGL 1 (translation block), zGL2 (splicing block) or control MO were scored at $24 \mathrm{hpf}$ for occurrence of a severe mutant phenotype defined by lack of clear somite boundaries and significant shortening of the embryo. The results are shown graphically as a mean percentage of total embryos injected $\pm S D$ of three experiments, with a minimum of $50 \mathrm{em}$ bryos injected per MO dose per experiment. Both zGL1 and zGL2 were equivalent in mediating the mutant effect which was not observed with controlMOs. (B) The efficiency and persistence of the splice-blocking MO zGL2 were confirmed using $R T-P C R$. Following injection of $10 \mathrm{ng}$ of $z G L 2$, but not control MO, a smaller gitrl amplification product, diagnostic for the absence of exon 2, was obtained. The effect of zGL2 on splicing remained fully effective to at least $18 \mathrm{hpf}$. (C) To confirm the omission of exon 2, transcripts were cloned from zGL2 and control MO injected embryos and a total of 24 control and 48 $z G L 2$ derived clones sequenced. All of the $z G L 2$ derived clones displayed exon 1-3 splicing. This mis-splicing introduced a frameshift mutation such that the TNF family domain region, underlined, would be replaced by an alternative, tuncated $C$-terminus, indicated in blue. All of the control clones contained the full-length gitrl sequence. (D-R) Embryos injected with control, zGL2 and zGR1 MOs were compared from early gastrula through late segmentation stages. During gastrulation the blastoderm margin was poorly defined at the shield stage (I) in zGL2 morphants and did not advance to cover the whole of the yolk cell ( J). Embryos tended to become mildly deformed during epiboly and the dorsal thickening and ventral thinning that is characteristic of late gastrulation was mispositioned in approximately $50 \%$ of the embryos as indicated by the arrow in (J). In contrast both control $(\mathbf{D}, \mathbf{E})$ and zGR1 $(\mathbf{N}, \mathbf{O})$ MO-injected embryos remained phenotypically normal during gastrulation. The first somite boundary was clearly delineated in control (arrowhead in (F)) and zGR1 (arrowhead in (P)) but not in zGL2 morphant (K) embryos. Abnormal somite shape, optic primordium and head shape can be seen in (L). By late segmentation, zGL2 MO-injected embryos were significantly shorter with abnormal yolk extensions and abnormally-shaped somites (M). A magnified view of the posterior somites clearly shows the normal chevron shape in controls (S) and the abnormal shape and less-clearly defined boundaries in zGL2 morphants (T). (Equivalent phenotypes were also observed using the zGL1 and zGR2, data not shown). (U) Control or (V) gitrl morphants were probed at the tail-bud stage $(10 \mathrm{hpf})$ using antisense RNA probes specific for hgg1 and dlx3. Double-headed arrows indicate the increased width of the $\mathrm{dl} \times 3$ expression domain in gitrl morphants relative to control embryos. Data are representative of embryos from at least three independent experiments in which a minimum of thirty embryos per MO per experiment were injected.

gitr/morphants have reduced cell movement during gastrulation.

Co-injection of synthetic capped gitrl mRNA was used to demonstrate the specificity of the MO knockdowns and as little as 100 pg of mRNA was sufficient to prevent the morphant phenotype, while control mRNA had no effect (Fig. 4).

\section{Strain-dependent enhancement of the gitrl morphant pheno- type}

The initial MO knockdown experiments were carried out on embryos from the wild type $A^{*}$ zebrafish line. When these experiments were repeated using wild type TL embryos, a more extreme form of the phenotype emerged (Fig. 5). In both the translation block and splice-site gitr/MO-injected $\mathrm{AB}^{*}$ embryos, the blastoderm margin advanced more slowly than in controls and failed to reach its full extent by the end of epiboly (Fig. 5 G-H). In
TL embryos, this became more marked as the embryo contracted in on itself (Fig. $5 \mathrm{~J}-\mathrm{L}$ ), eventually pinching off a segment of yolk and dying (Fig. 5L). Sequencing the genomic DNA of ten individual $A B^{*}$ and $T L$ fish embryos from stocks used in our experiments revealed no polymorphisms in the $z G L 1$ target region, suggesting that it is other differences within the genetic background of these two fish lines that ultimately underlie the differential severity of the gitr/-MO-induced morphant phenotype.

\section{Linking Gitrl to the Stat3 signalling pathway during early development}

Activation of the Stat3 pathway is required for cell movements in zebrafish embryogenesis (Yamashita et al., 2002), raising the possibility that the normal function of Gitrl may include the modulation of Stat3 phosphorylation. We assayed for levels of the 


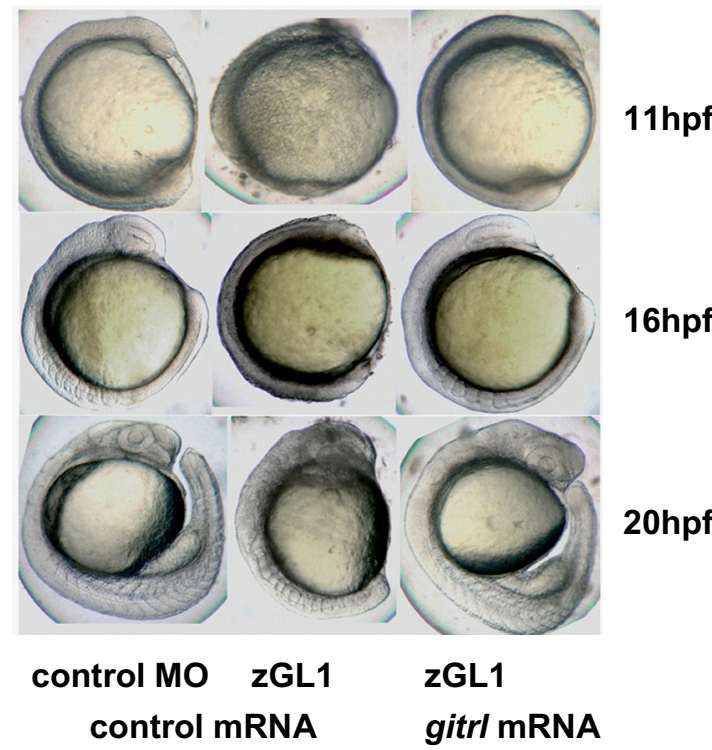

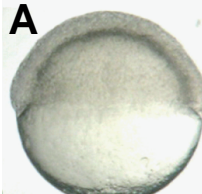
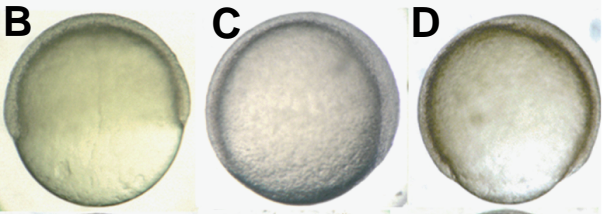

control TL
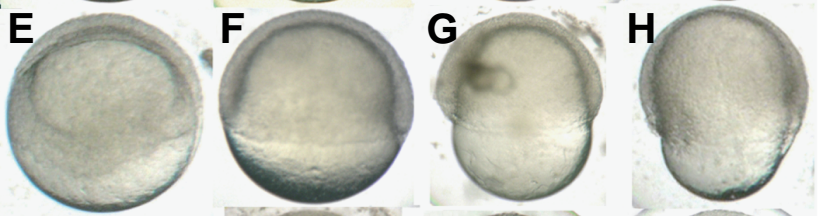

zGL1

$A B^{*}$
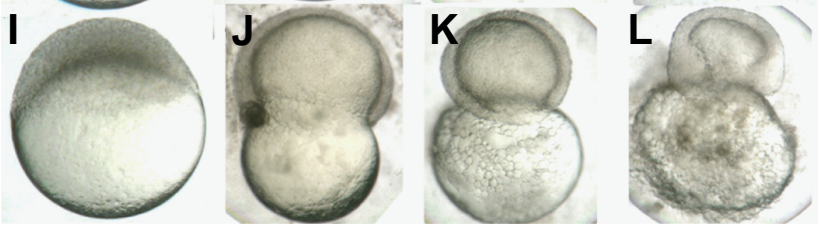

zGL1

TL

$7 \mathrm{hpf}$

8hpf

9hpf

Fig. 4 (Left). Rescue of the MO-induced gitrl morphant phenotype by co-injection of synthetic capped gitrl mRNA. Co-injection of synthetic capped mRNA and rescue of a normal phenotype was used to confirm that the MO-induced gitrl phenotype was specific and not due to off-target effects. Results with zGL1 (translation block) are shown, although equivalent rescue was also obtained for zGL2 (splice-block). The data are representative of embryos from at least three independent experiments in which a minimum of thirty embryos per MO per experiment were injected.

Fig. 5 (Right). Strain-dependent penetrance of the MO-induced gitrl morphant phenotype. Embryos of the (A-D, I-L) TL and (E-H) $A B^{*}$ strains were injected with 10 ng of either (A-D) control 1 or (E-L) zGL 1 MO. In zGL 1-injected AB* embryos advancement of the blastoderm margin was delayed and incomplete (F-H), but these embryos successfully completed epiboly and survived through the segmentation period (see Fig. 3). In contrast, zGL 1injected TL embryos could not complete epiboly, and demonstrated a progressive contraction of the embryos around the yolk (J-L)

activated, phosphorylated form of Stat3 protein by western blot and observed strong phosphorylation of Stat3 during the first 19 hours of development (Fig. 6A). Importantly, levels of phosphorylated Stat3 were decreased at all time points in the gitr/morphants. If one function of Gitrl is to modulate Stat3 signalling during embryogenesis, we hypothesised that inhibitors of this pathway might partially phenocopy the gitr/knockdown. As observed in gitr/morphants, treatment of 2-4 hpf embryos with cucurbitacin I (data not shown) or stattic (Fig. 6 G-K) resulted in delayed and incomplete advancement of the blastoderm margin (Fig. 6G) and indistinct somite boundaries (Fig. $6 \mathrm{H}-\mathrm{K}$ ). We observed evidence of strain differences in response to Stat3 inhibition, with mortality

Fig. 6. Identification of Stat3 phosphorylation as a possible mediator of Gitrl effects during embryogenesis. (A) Total protein lysates were generated from embryos injected with $10 \mathrm{ng}$ of control1 (C) or zGL 1 (MO) and harvested at 6, 8, 11 and $19 \mathrm{hpf}$. The lysates were analysed by western blotting using antibodies specific for phosphorylated (P-Stat3) and total (Stat3) Stat3 content. Embryos $\left(A B^{*}\right)$ treated with (B-F) DMSO or (G-K) $5 \mu \mathrm{M}$ of stattic at 2-4 hpf were compared for phenotypic overlap with the gitrl morphant phenotype shown in figure 3. Stattic-treated embryos displayed incomplete blastoderm margin advancement (arrowhead in (G)) compared to controls (arrowhead in (B)), and indistinct somite boundary formation (H-K) similar to that seen in gitrl morphants. (L) TL embryos were more sensitive than $A B^{*}$ embryos to Stat3 inhibition. (M) TL embryos treated with a low dose of zGL1 (2.5 ng) were additionally treated with either DMSO or $2 \mathrm{mM}$ of stattic at $2 \mathrm{hpf}$. Embryos that received low-dose MO plus DMSO were viable, but showed incomplete blastoderm margin advancement at $75 \%$ epiboly, while embryos treated with low-dose zGL 1 in addition to treatment with stattic showed a lethal phenotype.
A $\quad 6 \mathrm{hpf} 8 \mathrm{hpf} 11 \mathrm{hpf} 19 \mathrm{hpf}$ C MO C MO C MO C MO
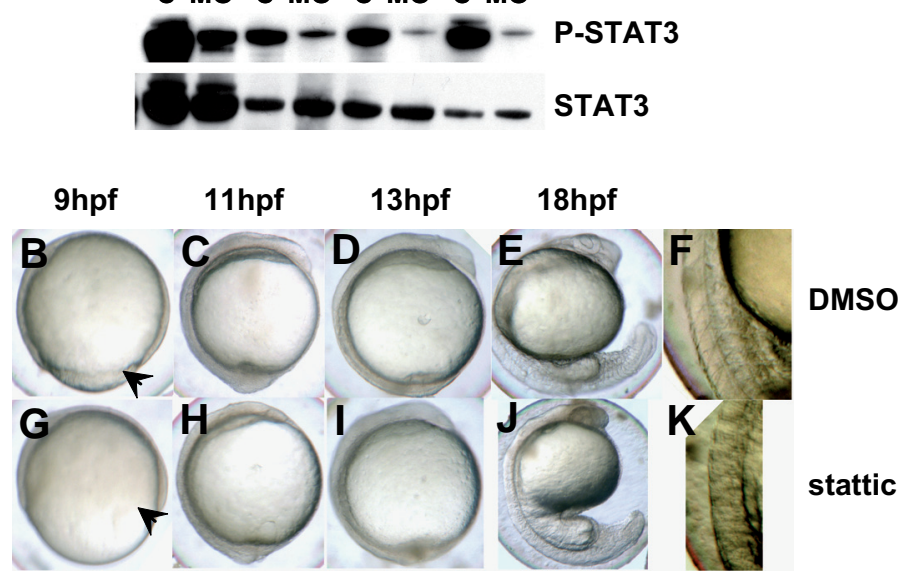

L

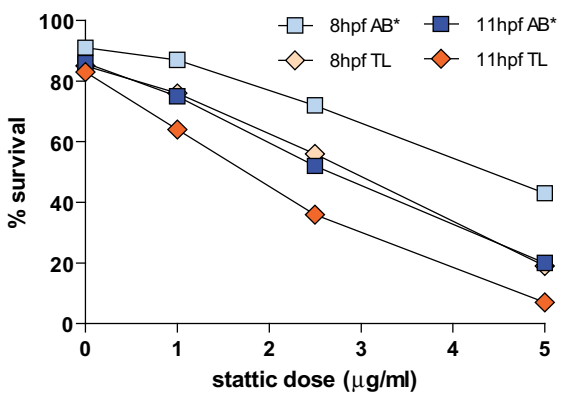

M

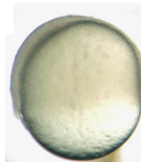

zGL1+ DMSO

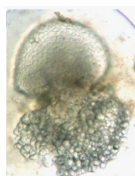

zGL1+ stattic 
to increasing concentrations of stattic higher among TL embryos than $\mathrm{AB}^{*}$ embryos at both 8 and $11 \mathrm{hpf}$ (Fig. 6L). Additionally, treatment with stattic increased the severity of the morphant phenotype in TL embryos given low doses of gitr/MO (Fig. 6M). Although Stat3 is essential for cell movement during gastrulation (Yamashita et al., 2002), subsequent to gastrulation and somitogenesis Stat3 and gitr/MO-induced morphants display different phenotypes, suggesting that while Gitrl may act upon Stat3 during early embryogenesis, decreased Stat3 signalling cannot fully account for the effects of gitrl knockdown.

Stat3 regulates expression of the zinc transporter Liv1, the

A
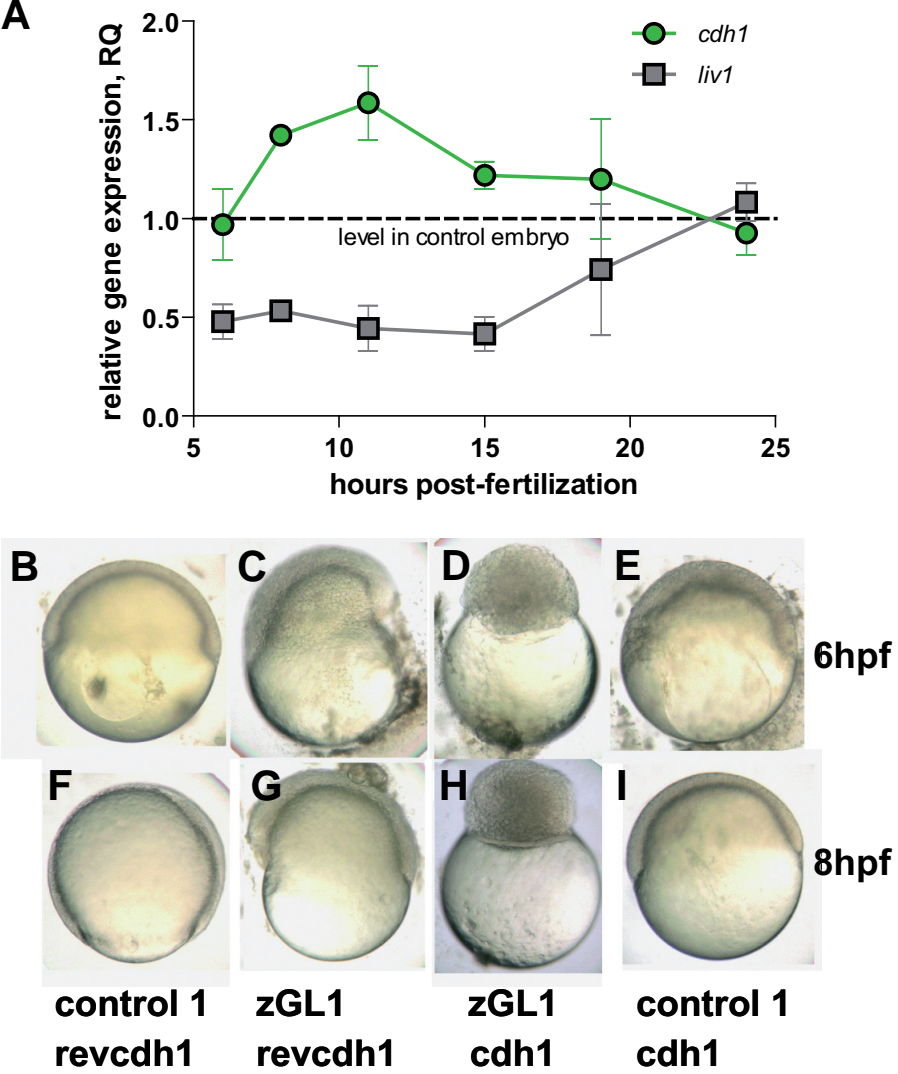

Fig. 7. Gitrl and the Stat3-Liv1-Cdh1 axis. (A) Transcription of liv1 and cdh1 were assessed by real-time PCR at various time points during gastrulation and segmentation in embryos injected with $10 \mathrm{ng}$ of either control1 or zGL1. Values were normalised to ef1 $\alpha$ and levels in zGL1 injected embryos expressed relative to levels detected in control-injected embryos using the $\Delta \Delta C$ t method (ABI Prism 7700 Sequence Detection System, user bulletin no. 2). Mean values $\pm S D$ of three independent experiments are shown. Embryos injected with either $(\mathbf{B}, \mathbf{F}) 10 \mathrm{ng}$ of control $1+2.5 \mathrm{ng}$ of cdh $1 \mathrm{MO}$ control (revcdh1), (C, G) $10 \mathrm{ng}$ of $z G L 1+2.5 \mathrm{ng}$ revcdh1, (D,H) $10 \mathrm{ng}$ of $z G L 1+2.5 \mathrm{ng}$ of cdh1 $\mathrm{MO}$ (cdh1) or (E,I) $10 \mathrm{ng}$ of control $1+2.5$ ng of cdh 1 were observed at (B-E) 6 and (F-I) 8 hpf. Embryos treated with control MOs only were indistinguishable from wild-type embryos. Those treated with the zGL 1 plus revcdh1 showed the characteristics of gitrl morphants previously described, and embryos injected with cdh1 MO plus control1 resembled the avalanche mutant (Kane et al., 1996). Surprisingly, embryos co-injected with both gitrl and cdh1 MOs had a novel phenotype characterized by arrest during the blastula period and a complete failure to enter epiboly. The phenotype shown in (D) and $\mathbf{( H )}$ persisted until the embryos died at approximately $15 \mathrm{hpf}$. activity of which in turn regulates nuclear localisation of the zincfinger protein Snail (Yamashita et al., 2004), which itself represses transcription of the cell adhesion molecule Cdh1 (Cano et al., 2000). In gitr/morphants, we found normal cdh1 mRNA levels prior to $5 \mathrm{hpf}$, but observed decreased liv1 expression and increased cdh 1 expression between 6 and 19 hpf (Fig. 7A). We wondered if the gastrulation-related effects of gitrl knockdown were due to increased $c d h 1$, and asked if knocking down $c d h 1$ by $\mathrm{MO}$ could at least partially rescue the gitr/MO-induced morphant phenotype (Fig. $7 \mathrm{~B}-\mathrm{I}$ ). To our surprise, co-injection of both gitrl and cdh $1 \mathrm{MOs}$ led to early arrest, with complete failure to begin gastrulation (Fig. $7 \mathrm{D}, \mathrm{H}$ ). As coht expression levels in gitrl morphants appeared normal prior to $5 \mathrm{hpf}$, one possibility is that Gitrl has a Cdh1-dependent function prior to the onset of gastrulation, and only later in development affects the Stat3-Liv1-Cdh1 axis.

\section{Discussion}

GITRL and GITR play an important role in the regulation of immune responses in mammals with signalling through GITR increasing resistance to certain tumours and infections, as well as limiting the ability of regulatory cells to suppress the immune response. While Gitr knockout mice are viable, and display altered $\mathrm{T}$ cell, inflammatory and anti-microbial responses, we found that loss of GITRL was strain-dependently lethal in early embryogenesis in mice (manuscript in preparation). The optical transparency of developing zebrafish offered a unique opportunity to investigate this embryonic lethal effect further, and we consequently sought to identify zebrafish GITRL and GITR homologues.

Putative gitrl and gitr exons were identified within zebrafish genomic databases by homology and the corresponding fulllength transcripts cloned. Assignment of both genes was supported by phylogenetic clustering of the cloned nucleotide coding regions with well-characterised mammalian homologues. While expression of zebrafish gitrwas detected in adult kidney, spleen, thymus and gut, it was not detected during early development, and embryos developed normally in the presence of gitrspecific MOs. The gitr gene maps to chromosome 6 on the current zebrafish genome build (assembly Zf7). There is no obvious synteny with mouse or human gitr, however the lack of impact of gitr-targeted MOs and absence of mRNA expression in early embryogenesis are consistent with the viable status of Gitr knockout mice, and the documented role of GITR in controlling the mammalian immune response (Agostini et al., 2005, Cuzzocrea et al., 2006, Ronchetti et al., 2002, Shimizu et al., 2002, Tone et al., 2003). MOs targeted against gitrl gave rise to a complex developmental phenotype displaying effects on multiple systems. Approximately $15-20 \%$ of MOs are known to show off-target effects, which can indeed include somite abnormalities similar to those observed here, however two independent gitr/MOs, one blocking translation and the other blocking splicing, both gave rise to the same developmental phenotype and the gitr/ morphant phenotype was specifically rescued by complementation with synthetic capped gitrl, but not control mRNA. Thus the complex morphant phenotype observed here appears to be gitr-specific and, coupled with the multiphasic and localised expression pattern observed in the first $24 \mathrm{hpf}$, suggests that zebrafish Gitrl has 
a role at more than one point in early embryogenesis.

Although none of the reported TNF or TNFR superfamily members knocked out in mice to date have been associated with an embryonic lethal phenotype, family members have been implicated in development, for example, of the secondary lymphoid system (Fu and Chaplin, 1999). RANKL has been shown to regulate osteoclastogenesis, in addition to roles in lymph-node organogenesis and lymphocyte development (Kong et al., 1999), while mutations in EDA have indicated involvement in skin, hair and tooth morphogenesis (Ferguson et al., 1997, Kere et al., 1996). We entered into this investigation seeking insight into the embryonic lethal phenotype that we had encountered in the absence of GITRL in mice. Our data now suggest the possible involvement of an alternative receptor, or else a role for reverse signalling through the ligand (Sun and Fink, 2007). It is not uncommon for TNFSF ligands to bind more than one receptor, however, it has been shown that human and mouse GITRL do not bind to any other currently known mammalian TNFRSF members (Bossen et al., 2006), and so if there is an alternative GITRL receptor involved in murine embryonic development then it has not yet been identified. The phenomenon of reverse signalling has been described for some TNFSF members and was postulated as a mechanism by which soluble GITR could enhance secretion of matrix metalloproteinase 9 (MMP9) from macrophages, although this effect has more recently been shown to be due to blockade of GITR-induced MMP-9 inhibition (Suvas et al., 2005). None of the GITRL sequences have the cytoplasmic casein kinase I motif, SXXS-, that has been associated with reverse signalling (Sun and Fink, 2007).

We have shown that zebrafish Gitrl influences Stat3 phosphorylation, and raised the possibility that decreased Stat3 phosphorylation in the ligand morphants is responsible for a co-ordinate decrease in liv1 and increase in cdh1 expression, leading to increased cell-cell adhesion affecting cell movement (Yamashita et al., 2004). This could be sufficient to account for the slow and incomplete nature of gastrulation in the morphants. Nevertheless, our experiments with Stat3 inhibition, as well as published work on stat3 MO effects (Yamashita et al., 2002), show that decreased Stat3 signalling does not fully account for the observed gitrl morphant phenotype. This suggests that there are other mechanisms of development affected, and the kinetics of gitr/expression, with multiple expression peaks, would imply independent effects at different time-points.

We observed strain dependent differences in the affects of gitrl knockdown in zebrafish. There were no nucleotide polymorphisms in the relevant MO target sequence, eliminating this as an explanation of the difference. There was evidence of differing sensitivity to Stat3 inhibition, raising the possibility that constitutive differences in Stat3 signalling exist between the two test strains. That the effects of Gitrl loss can be enhanced on specific genetic backgrounds offers the possibility of future genetic and small molecule screens to identify modulators of this pathway in early development.

Previous mining of teleost genomes described fourteen distinct TNFSF member genes, but failed to identify clear orthologs of four genes encoding members involved in mammalian $\mathrm{T}$ cell activation and homeostasis, namely OX40L, CD27L, CD30L and GITRL (Glenney and Wiens, 2007). In humans and mice, the GITRL gene is located on chromosome 1, flanked by genes encoding FASL and OX40L. Genes encoding LIGHT, CD27L and 4-1BBL form a separate TNFSF cluster on human chromosome $19 /$ mouse chromosome 17. Here, we assigned zebrafish gitrl based on homology and protein alignment data, which was supported by phylogenetic cluster analysis of nucleotide coding regions (Fig. 1). We note that this sequence has been placed on the current zebrafish genome build (assembly Zf7) downstream of light, in a position consistent with mammalian 4-1BBL. Interestingly, the gene annotated as zebrafish light did not group with mammalian L/GHThomologues in the nucleotide based phylogenetic analysis shown in Fig. 1C. It did move to the LIGHT grouping when the phylogeny was repeated using protein data (data not shown). The zebrafish Gitrl also moved to group with mammalian 4-1BBL homologues in this analysis; although by pairwise alignment the zebrafish Gitrl shared lower identity with human and mouse 4-1BBL, than the GITRL homologues (13 versus $18 \%$ ), and the reverse signalling casein kinase I motif conserved in mammalian 4-1BBL homologues was absent from zebrafish Gitrl (Sun and Fink, 2007), both features failing to support this alternate gene assignment. Furthermore, while embryonic defects have not been reported in mice lacking 4-1BBL (DeBenedette et al., 1999), both the absence of GITRL in mice and knockdown of the proposed gitr/in zebrafish generated a strain dependant embryonic lethal phenotype.

In general terms the zebrafish immune system has proved remarkably similar to that of mammals, and is being developed to model human disease (Meeker and Trede, 2008). Here we have identified and cloned TNF/TNFRSF members related by homology and phylogenetic analysis to mammalian GITRL and GITR. We have demonstrated a multiphasic pattern of gitrlexpression in the first $24 \mathrm{hpf}$, and that knockdown of this expression gives rise to a complex phenotype to reveal a critical requirement for Gitrl in early embryogenesis, at the very least involved in gastrulation. These effects are mediated prior to the expression of Gitr, its only currently known receptor in mammals. Our studies implicating Gitrl in the control of Stat3 signalling during early zebrafish development provide an initial platform for future studies to determine if Gitrl can also regulate Stat3 signalling in mouse development. In conclusion our data has established that Gitrl, which came to prominence because of its role in regulation of the mammalian immune system, also has a previously unappreciated role in vertebrate embryonic development mediated independently of Gitr.

\section{Materials and Methods}

\section{Fish stocks}

Adult zebrafish of the $\mathrm{AB}^{*}$ and TL strains were maintained on a $14 \mathrm{hr} /$ $10 \mathrm{hr}$ light/dark cycle at $28.5^{\circ} \mathrm{C}$. Embryos were staged according to (Kimmel et al., 1995).

Human and mouse GITRL and GITR transcripts were used to query the zebrafish genome using blastn. Putative coding sequences were assembled from the genomic data and full-length coding sequences PCR-amplified and cloned to PGEM-T easy (Promega) using primers: gitrl

5'-TCACTACTGCATCATGTCTCTGT-3' and

5'-TGACTGAGATTTAGCCAAGCTTGATGACA-3', and

gitr

ACGCATTGGTAATGCACCACAGTG-3' and

5'-AGGAAAGCTGTGGTCACAGCAGT-3'. 
Multiple clones were sequenced. Sequence alignments were performed using Jalview 2.4 (Waterhouse et al., 2009).

\section{Phylogenetic analysis}

Closely related superfamily members were identified using human and mouse GITRL and GITR and the newly identified zebrafish sequences to query the nr database by tblastn. For the ligand, TNFSF5, 7, 10 and 11 were included on the basis of sequence homology, TNFSF4, 6,9 and 14 by genomic proximity and ectodysplasin and EIGER were also included. For the receptor, TNFRSF4, 5, 9, 11 and 14 were included on the basis of tblastn and TNFRSF 6 and 7 to complement the ligand analysis. Species homologues were identified, but only those with synteny information supporting their assignment were included (at least one flanking gene identified). Multiple sequence FASTA files of ligand and receptor nucleotide coding and protein sequences were compiled and analysed using the 'one-click' phylogeny application, at http:// www.phylogeny.fr/ (without GBlock refinement) (Dereeper et al., 2008).

\section{RNA isolation and real-time PCR}

RNA was extracted from adult tissues or whole embryos using the SV Total RNA Isolation System (Promega) and reverse transcribed using the Stratascript First Strand Synthesis System (Stratagene). Real-time PCR was performed as previously described (Nolan et al., 2004) using the following primers and probes:

zgitr/primers

5'-CTAATGACATTCACGCCAAACTG-3' and

5'-CAGCTGTCCCTCTCCTTTGATG-3' and

probe

5'FAM-TTCCACTGCAGGAGCTCAACATCTGTGT-3'; zgitrprimers

5'-GCTGTAAAGCCTGTCCTGCAT-3' and

5'-GAAGGGCATTTCTCACACAGACT-3' and

probe

5'FAM-CCTATATCGGCATGTTCACAAGACAGGACA-3';

zef1 $\alpha$ primers

5'-CCACGTCGACTCCGGAAA-3' and

5'-CGATTCCACCGCATTTGTAGA-3' and

probe 5'VIC-TCCACCACCACCGGCCATCTG-3';

zliv1 primers

5'- GCTGAACGCGCTTACTTTCG -3' and

5'- CAGCAGTGCCAGTGACATCAC -3' and

probe

5'FAM- CGTGGACTCCCGGTTGCCAATATG -3';

$z c d h 1$ primers

5'- CTGCTATTGCTTGCTCTTCTTCTG -3' and

5'- CATCATAATAGTAGATGTTGTCCCGTACA -3' and

probe

5'FAM- TCCTGCGGAGGAAAAGCAACTCCAA -3'.

For each of three biological replicates, embryos were pooled from timed mating of 8-10 pairs of fish and collected at the indicated time-points over a contiguous period.

\section{In situ hybridisation}

Whole-mount in situ hybridisation was carried out as described by (Thisse and Thisse, 2008). Antisense RNA probes were transcribed from linearised templates using T3, T7, or SP6 RNA polymerases (Promega) in the presence of digoxigenin (DIG)-labelled nucleotides (Roche). DIG was detected using anti-DIG-alkaline phosphatase Fab and BM-Purple (Roche). For the gitr/candidate the probe was synthesised from the fulllength coding sequence. The probe constructs for $d / x 3$ and $h g g 1$ were a kind gift of Jeroen den Hertog (Utrecht, The Netherlands).

\section{Morpholino oligonucleotides (MOs)}

Antisense MOs were designed by and obtained from GeneTools (Philomath, OR)
zGL1 5'-TTCGGCAGACAGAGACATGATGCAG-3' (translation block); zGL2 5'-TTAAATGGTGACTTACAGTTTGGCG-3' (splice block exon1exon2);

control 15'-GACGTAGTACAGAGACAGACGGCTT-3' (reverse of zGL1); control 2 5'-GCGGTTTGACATTCAGTGGTAAATT-3' (reverse of zGL2); zGR1 5'-TGTTCATTCTGTCACTGTGGTGCAT-3' (translation block); and zGR2 5'-GATATAGGATACTCGCCTGTACAAT-3' (splice block exon1exon2).

The antisense splicing-blocking $\mathrm{MO}$ for e-cadherin, cdh1, was that described by (Shimizu et al., 2005):

5 2-AAAGTCTTACCTGAAAAAGAAAAAC-3 2. The reverse sequence was used as control.

Stock solutions, $10 \mu \mathrm{g} / \mu \mathrm{L}$ of each MO, were prepared in Danieau buffer, and 1 to $15 \mathrm{ng}$ of stock solution, or specified dilutions, injected into 1-4 cell-stage embryos using a Picospritzer II microinjector (Parker Instrumentation). The efficiency of the splice-blocking zGL2 MO was monitored by RT-PCR using the full-length gitr/primers described above. Efficiency of the zGR2 splice-blocking MO could not be determined because gitr could not be amplified from either control or experimental groups in the first two days of development.

\section{Capped mRNA synthesis}

Capped, polyadenylated mRNA for zebrafish gitr/and for mouse Tnf (control RNA) was in vitrotranscribed from pGEM-T easy plasmids using SP6 or T7 mMessage mMachine kits (Ambion, Austin, TX) and injected into embryos at the one-cell stage. One replicate of the rescue experiment was done blinded to eliminate the possibility of observer bias.

\section{Western blotting}

Protein lysates were separated on denaturing SDS-PAGE gels, transferred to a PDVF membrane and probed using anti-zebrafish phosphoStat3 (MBL International), or anti-Stat3 (Santa Cruz) primary, and peroxidase-conjugated secondary antibodies. Antibody binding was detected using ECL solutions (Pierce).

\section{Treatment of embryos with inhibitors}

Stock solutions of cucurbitacin I (Calbiochem) or stattic (Calbiochem) dissolved in DMSO were added at the stated concentrations to embryos in 6-well plates. Control embryos were treated with DMSO alone at the same concentrations.

\section{Acknowledgements}

We thank Jeroen den Hertog (Hubrecht Institute, Utrecht, Netherlands) for in situ hybridization plasmids, Masahiko Hibi (RIKEN, Japan) for experimental advice and plasmids, Nick Temperley (MRC Human Genetics Unit \& The University of Edinburgh, UK) and Fernando Martinez (SWDSOP, Oxford, UK) for support regarding bioinformatics, Elizabeth Robertson (SWDSOP, Oxford, UK) for advice on the manuscript, and Roger Patient and his laboratory (WIMM, Oxford, UK) for experimental discussion. This work was supported by an MRC Programme Grant, HW, and an MRC Career Development Award, EEP.

\section{References}

AGOSTINI, M., CENCI, E., PERICOLINI, E., NOCENTINI, G., BISTONI, G. VECCHIARELLI, A. and RICCARDI, C. (2005). The glucocorticoid-induced tumor necrosis factor receptor-related gene modulates the response to Candida albicans infection. Infect Immun 73: 7502-7508.

BOSSEN, C., INGOLD, K., TARDIVEL, A., BODMER, J.L., GAIDE, O., HERTIG, S. AMBROSE, C., TSCHOPP, J. and SCHNEIDER, P. (2006). Interactions of tumor necrosis factor (TNF) and TNF receptor family members in the mouse and human. J Biol Chem 281: 13964-13971.

CANO, A., PEREZ-MORENO, M.A., RODRIGO, I., LOCASCIO, A., BLANCO, M.J., DEL BARRIO, M.G., PORTILLO, F. and NIETO, M.A. (2000). The transcription factor snail controls epithelial-mesenchymal transitions by repressing $\mathrm{E}$-cadherin 
expression. Nat Cel/ Bio/2: 76-83.

CUZZOCREA, S., NOCENTINI, G., DI PAOLA, R., AGOSTINI, M., MAZZON, E., RONCHETTI, S., CRISAFULLI, C., ESPOSITO, E., CAPUTI, A.P. and RICCARDI, C. (2006). Proinflammatory role of glucocorticoid-induced TNF receptor-related gene in acute lung inflammation. $J$ Immuno/177: 631-641.

DEBENEDETTE, M.A., WEN, T., BACHMANN, M.F., OHASHI, P.S., BARBER, B.H., STOCKING, K.L., PESCHON, J.J. and WATTS, T.H. (1999). Analysis of 4-1BB ligand (4-1BBL)-deficient mice and of mice lacking both 4-1BBL and CD28 reveals a role for 4-1BBL in skin allograft rejection and in the cytotoxic $T$ cell response to influenza virus. $J /$ mmuno/ 163: 4833-4841.

DEREEPER, A., GUIGNON, V., BLANC, G., AUDIC, S., BUFFET, S., CHEVENET, F., DUFAYARD, J.F., GUINDON, S., LEFORT, V., LESCOT, M. et al. (2008). Phylogeny.fr: robust phylogenetic analysis for the non-specialist. Nucleic Acids Res 36: W465-469.

FERGUSON, B.M., BROCKDORFF, N., FORMSTONE, E., NGYUEN, T., KRONMILLER, J.E. and ZONANA, J. (1997). Cloning of Tabby, the murine homolog of the human EDA gene: evidence for a membrane-associated protein with a short collagenous domain. Hum Mol Genet 6: 1589-1594.

FRATTINI, A., VEZZONI, P., VILLA, A. and SOBACCHI, C. (2007). The Dissection of Human Autosomal Recessive Osteopetrosis Identifies an Osteoclast-Poor Form due to RANKL Deficiency. Cell Cycle 6: 3027-3033.

FU, Y.X. and CHAPLIN, D.D. (1999). Development and maturation of secondary lymphoid tissues. Annu Rev Immuno/17: 399-433.

GLENNEY, G.W. and WIENS, G.D. (2007). Early diversification of the TNF superfamily in teleosts: genomic characterization and expression analysis. $J$ Immuno/178: 7955-7973.

KANE, D.A., HAMMERSCHMIDT, M., MULLINS, M.C., MAISCHEIN, H.M., BRAND, M., VAN EEDEN, F.J., FURUTANI-SEIKI, M., GRANATO, M., HAFFTER, P., HEISENBERG, C.P. et al. (1996). The zebrafish epiboly mutants. Development 123: 47-55.

KERE, J., SRIVASTAVA, A.K., MONTONEN, O., ZONANA, J., THOMAS, N., FERGUSON, B., MUNOZ, F., MORGAN, D., CLARKE, A., BAYBAYAN, P. etal. (1996). X-linked anhidrotic (hypohidrotic) ectodermal dysplasia is caused by mutation in a novel transmembrane protein. Nat Genet 13: 409-416.

KIMMEL, C.B., BALLARD, W.W., KIMMEL, S.R., ULLMANN, B. and SCHILLING, T.F. (1995). Stages of embryonic development of the zebrafish. Dev Dyn203: 253-310.

KONG, Y.Y., YOSHIDA, H., SAROSI, I., TAN, H.L., TIMMS, E., CAPPARELLI, C., MORONY, S., OLIVEIRA-DOS-SANTOS, A.J., VAN, G., ITIE, A. et al. (1999). OPGL is a key regulator of osteoclastogenesis, lymphocyte development and lymph-node organogenesis. Nature 397: 315-323.

KRENS, S.F., HE, S., LAMERS, G.E., MEIJER, A.H., BAKKERS, J., SCHMIDT, T., SPAINK, H.P. and SNAAR-JAGALSKA, B.E. (2008). Distinct functions for ERK1 and ERK2 in cell migration processes during zebrafish gastrulation. Dev Bio/319: 370-383.
MEEKER, N.D. and TREDE, N.S. (2008). Immunology and zebrafish: spawning new models of human disease. Dev Comp Immuno/32: 745-757.

MORENO, E., YAN, M. and BASLER, K. (2002). Evolution of TNF signaling mechanisms: JNK-dependent apoptosis triggered by Eiger, the Drosophila homolog of the TNF superfamily. Curr Bio/12: 1263-1268.

NOLAN, K.F., STRONG, V., SOLER, D., FAIRCHILD, P.J., COBBOLD, S.P., CROXTON, R., GONZALO, J.A., RUBIO, A., WELLS, M. and WALDMANN, $\mathrm{H}$. (2004). IL-10-conditioned dendritic cells, decommissioned for recruitment of adaptive immunity, elicit innate inflammatory gene products in response to danger signals. J Immuno/172: 2201-2209.

RONCHETTI, S., NOCENTINI, G., RICCARDI, C. and PANDOLFI, P.P. (2002). Role of GITR in activation response of T lymphocytes. Blood 100: 350-352.

SHIMIZU, J., YAMAZAKI, S., TAKAHASHI, T., ISHIDA, Y. and SAKAGUCHI, S. (2002). Stimulation of CD25(+)CD4(+) regulatory T cells through GITR breaks immunological self-tolerance. Nat Immuno/3: 135-142.

SHIMIZU, T., YABE, T., MURAOKA, O., YONEMURA, S., ARAMAKI, S., HATTA, K., BAE, Y.K., NOJIMA, H. and HIBI, M. (2005). E-cadherin is required for gastrulation cell movements in zebrafish. Mech Dev122: 747-763.

SUN, M. and FINK, P.J. (2007). A new class of reverse signaling costimulators belongs to the TNF family. J Immuno/179: 4307-4312.

SUVAS, S., KIM, B., SARANGI, P.P., TONE, M., WALDMANN, H. and ROUSE, B.T. (2005). In vivo kinetics of GITR and GITR ligand expression and their functional significance in regulating viral immunopathology. J Viro/79: 11935-11942.

THISSE, C. and THISSE, B. (2008). High-resolution in situ hybridization to wholemount zebrafish embryos. Nat Protoc 3: 59-69.

TONE, M., TONE, Y., ADAMS, E., YATES, S.F., FREWIN, M.R., COBBOLD, S.P. and WALDMANN, H. (2003). Mouse glucocorticoid-induced tumor necrosis factor receptor ligand is costimulatory for T cells. Proc Nat/Acad Sci USA 100: 15059-15064.

WATERHOUSE, A.M., PROCTER, J.B., MARTIN, D.M., CLAMP, M. and BARTON, G.J. (2009). Jalview Version 2-a multiple sequence alignment editor and analysis workbench. Bioinformatics 25: 1189-1191.

YAMASHITA, S., MIYAGI, C., CARMANY-RAMPEY, A., SHIMIZU, T., FUJII, R., SCHIER, A.F. and HIRANO, T. (2002). Stat3 Controls Cell Movements during Zebrafish Gastrulation. Dev Cel/2: 363-375.

YAMASHITA, S., MIYAGI, C., FUKADA, T., KAGARA, N., CHE, Y.S. and HIRANO, T. (2004). Zinc transporter LIVI controls epithelial-mesenchymal transition in zebrafish gastrula organizer. Nature 429: 298-302.

YE, H., PARK, Y.C., KREISHMAN, M., KIEFF, E. and WU, H. (1999). The structural basis for the recognition of diverse receptor sequences by TRAF2. Mol Cel/4: 321-330.

ZELENIKA, D., ADAMS, E., HUMM, S., GRACA, L., THOMPSON, S., COBBOLD, S.P. and WALDMANN, H. (2002). Regulatory T cells overexpress a subset of Th2 gene transcripts. J Immuno/168: 1069-1079. 


\section{Further Related Reading, published previously in the Int. J. Dev. Biol.}

See our recent Special Issue Epigenetics \& Development edited by Saadi Khochbin and Stefan Nonchev at:

http://www.ijdb.ehu.es/web/contents. php?vol=53\&issue=2-3

Expression of zinc transporter family genes in Dictyostelium

Nobuya Sunaga, Meri Monna, Nao Shimada, Mai Tsukamoto and Takefumi Kawata Int. J. Dev. Biol. (2008) 52: 377-381

A change in response to Bmp signalling precedes ectodermal fate Choice Chris T. Dee, Abigail Gibson, Andrea Rengifo, Shun-Kuo Sun, Roger K. Patient and Paul J. Scotting

Int. J. Dev. Biol. (2007) 51: 79 - 84

Evidence that the rat osteopetrotic mutation toothless (tl) is not in the TNFSF11 (TRANCE, RANKL, ODF, OPGL) gene

P R Odgren, N Kim, L van Wesenbeeck, C MacKay, A Mason-Savas, F F Safadi, S N Popoff, C Lengner, W van-Hul, Y Choi and S C Marks

Int. J. Dev. Biol. (2001) 45: 853-859

Tumor necrosis factor-alpha inhibits epithelial differentiation and morphogenesis in the mouse metanephric kidney in vitro

C M Cale, N J Klein, G Morgan and A S Woolf

Int. J. Dev. Biol. (1998) 42: 663-674

Distribution of TNF alpha-like proteins correlates with some regions of programmed cell death in the chick embryo

M A Wride, P H Lapchak and E J Sanders

Int. J. Dev. Biol. (1994) 38: 673-682

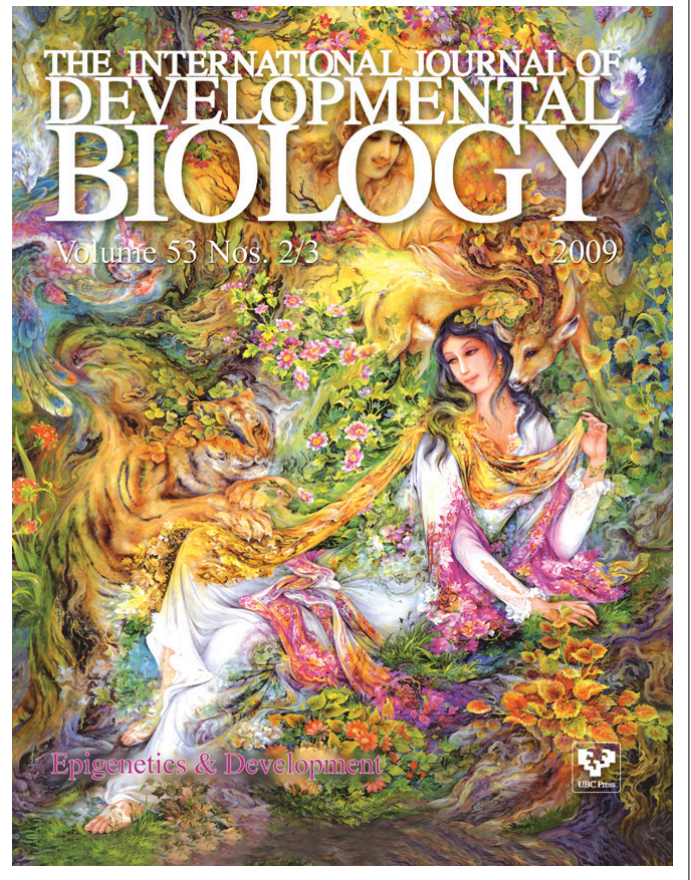

For all the latest on Pattern Formation research, see our latest Special Issue edited by C.-M. Chuong and M.K. Richardson.

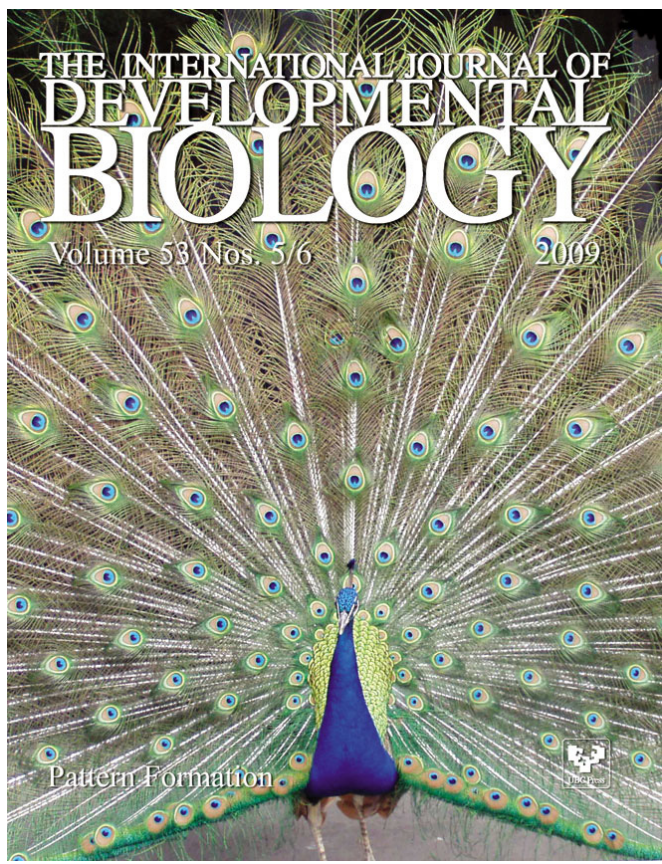

